\title{
A new architecture model for smart manufacturing: A performance analysis and comparison with the RAMI 4.0 reference model
}

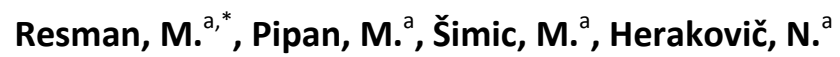 \\ ${ }^{a}$ Department of Manufacturing Technologies and Systems, Faculty of Mechanical Engineering, University of Ljubljana, \\ Ljubljana, Slovenia
}

\section{A B S T R A C T}

In this paper we proposed a new architectural model of the smart factory to allow production experts to make easier and more exact planning of new, smart factories by using all the key technologies of Industry 4.0. The existing complex reference architectural model of Industry 4.0 (RAMI 4.0) offers a good overview of the smart-factory architecture, but it leads to some limitations and a lack of clarity for the users. To overcome these limitations, we have developed a simple model with the entire and very simple architecture of the smart factory, based on the concept of distributed systems with exact information and the data flows between them. The proposed architectural model enables more reliable and simple modelling of the smart factory than the existing RAMI 4.0 model. Our approach improves the existing methodology for planning the smart factory and makes all the necessary steps clearer. At the end of the paper a comparison of the proposed architectural model LASFA (LASIM Smart Factory) with the existing RAMI 4.0 model was made. The developed LASFA model was already successfully implemented in the laboratory environment for building the demo centre of a smart factory.
\end{abstract}

\section{ARTICLE INFO}

Keywords:

Industry 4.0;

Smart manufacturing;

Smart factory;

Architectural model;

Reference architectural model;

RAMI 4.0

*Corresponding author: matevz.resman@fs.uni-lj.si (Resman, M.)

Article history:

Received 20 December 2018

Revised 24 April 2019

Accepted 7 May 2019

(c) 2019 CPE, University of Maribor. All rights reserved.

\section{References}

[1] Lu, H.-P., Weng, C.-I. (2018). Smart manufacturing technology, market maturity analysis and technology roadmap in the computer and electronic product manufacturing industry, Technological Forecasting and Social Change, Vol. 133, 85-94, doi: 10.1016/i.techfore.2018.03.005.

[2] Weber, C., Königsberger, J., Kassner, L., Mitschang, B. (2017). M2DDM - A maturity model for data-driven manufacturing, Procedia CIRP, Vol. 63, 173-178, doi: 10.1016/i.procir.2017.03.309.

[3] PwC. Industry 4.0 - Enabling digital operations, from https://i40-self-assessment.pwc.de/i40/landing/, accessed November 10, 2018.

[4] Impuls. Industrie 4.0-Readiness: Online-Selbst-Check für Unternehmen, from https://www.industrie40readiness.de/, accessed October 12, 2018.

[5] IHK. Industrie 4.0 Reifegrad - Selbstcheck für Unternehmen, from https://ihk-industrie40.de/selbstcheck/, accessed October 15, 2018.

[6] Anderl, R., Picard, A., Wang, Y., Fleischer, J., Dosch, S., Klee, B., Bauer, J. (2015). Leitfaden Industrie 4.0 - Orientirungshilfe zur Einführung in den Mittelstand, VDMA, Frankfurt am Main, Germany.

[7] Schweichhart, K. Reference architecture model Industrie 4.0 (RAMI 4.0), from https://ec.europa.eu/futurium/en/system/files/ged/a2-schweichhartreference architectural model industrie 4.0 rami 4.0.pdf, accessed November 27, 2018.

[8] Industrial Internet Consortium. Industrial internet reference architecture, from $\underline{\text { https://www.iiconsortium.org/ }}$ IIRA-1-7-ajs.pdf, accessed October 17, 2018. 
[9] Gröger, C., Kassner, L., Hoos, E., Königsberger, J., Kiefer, C., Silcher, S., Mitschang, B. (2016). The data-driven factory - Leveraging big industrial data for agile, learning and human-centric manufacturing, In: Proceedings of the 18th International Conference on Enterprise Information Systems, Rome, Italy, 40-52, doi: 10.5220/0005831500 400052.

[10] European Commission. Platforms for connected factories of the future, from http://ec.europa.eu/information society/newsroom/image/document/201548/workshop report platforms oct15 finaldocx 12361.pdf, accessed September 22, 2018.

[11] Weyrich, M., Ebert, C. (2016). Reference architectures for the internet of things, IEEE Software, Vol. 33, No. 1, 112-116, doi: 10.1109/MS.2016.20.

[12] Monostori, L., Váncza, J., Kumara, S.R.T. (2006). Agent-based systems for manufacturing, CIRP Annals, Vol. 55, No. 2, 697-720, doi: 10.1016/j.cirp.2006.10.004.

[13] Monostori, L., Kádár, B., Bauernhansl, T., Kondoh, S., Kumara, S., Reinhart, G., Sauer, O., Schuh, G., Sihn, W., Ueda, K. (2016). Cyber-physical systems in manufacturing, CIRP Annals, Vol. 65, No. 2, 621-641, doi: 10.1016/i.cirp. 2016.06.005.

[14] Kemény, Z., Beregi, R.J., Erdős, G., Nacsa, J. (2016). The MTA SZTAKI smart factory: Platform for research and project-oriented skill development in higher education, Procedia CIRP, Vol. 54, 53-58, doi: 10.1016/i.procir. 2016.05.060.

[15] Kemény, Z., Nacsa, J., Erdős, G., Glawar, R., Sihn, W., Monostori, L., Ilie-Zudor, E. (2016). Complementary research and education opportunities - A comparison of learning factory facilities and methodologies at TU Wien and MTA SZTAKI, Procedia CIRP, Vol. 54, 47-52, doi: 10.1016/i.procir.2016.05.064.

[16] Valckenaers, P., Van Brussel, H. (2005). Holonic manufacturing execution systems, CIRP Annals, Vol. 54, No. 1, 427-432, doi: 10.1016/S0007-8506(07)60137-1.

[17] Bagheri, B., Yang, S., Kao, H.-A., Lee, J. (2015). Cyber-physical systems architecture for self-aware machines in industry 4.0 environment, IFAC-PapersOnLine, Vol. 48, No. 3, 1622-1627, doi: 10.1016/j.ifacol.2015.06.318.

[18] Leitão, P., Colombo, A.W., Karnouskos, S. (2016). Industrial automation based on cyber-physical systems technologies: Prototype implementations and challenges, Computers in Industry, Vol. 81, 11-25, doi: 10.1016/ j.compind.2015.08.004.

[19] Bongaerts, L., Monostori, L., McFarlane, D., Kádár, B. (2000). Hierarchy in distributed shop floor control, Computers in Industry, Vol. 43, No. 2, 123-137, doi: 10.1016/S0166-3615(00)00062-2.

[20] Hussain, S., Jahanzaib, M. (2018). Sustainable manufacturing - An overview and a conceptual framework for continuous transformation and competitiveness, Advances in Production Engineering \& Management, Vol. 13, No. 3, 237-253, doi: 10.14743/apem2018.3.287.

[21] Vieira, A.A.C., Dias, L.M.S., Santos, M.Y., Pereira, G.A.B., Oliveira, J.A. (2018). Setting an industry 4.0 research and development agenda for simulation - A literature review, International Journal of Simulation Modelling, Vol. 17, No. 3, 377-390, doi: 10.2507/IJSIMM17(3)429.

[22] Zheng, P., Wang, H., Sang, Z., Zhong, R.Y., Liu, Y., Liu, C., Mubarok, K., Yu, S., Xu, X. (2018). Smart manufacturing systems for industry 4.0: Conceptual framework, scenarios, and future perspectives, Frontiers of Mechanical Engineering, Vol. 13, No. 2, 137-150, doi: 10.1007/s11465-018-0499-5.

[23] Zhang, Y., Zhang, G., Wang, J., Sun, S., Si, S., Yang, T. (2015). Real-time information capturing and integration framework of the internet of manufacturing things, International Journal of Computer Integrated Manufacturing, Vol. 28, No. 8, 811-822, doi: 10.1080/0951192X.2014.900874.

[24] Liu, Y., Xu, X. (2016). Industry 4.0 and cloud manufacturing: A comparative analysis, Journal of Manufacturing Science and Engineering, Vol. 139, No. 3, doi: 10.1115/1.4034667.

[25] Zezulka, F., Marcon, P., Vesely, I., Sajdl, O. (2016). Industry 4.0 - An introduction in the phenomenon, IFACPapersOnLine, Vol. 49, No. 25, 8-12, doi: 10.1016/j.ifacol.2016.12.002.

[26] Uslar, M., Hanna, S. (2018). Model-driven requirements engineering using RAMI 4.0 based visualization. In: Schaefer, I., Cleophas, L., Felderer, M. (eds.), Workshops at Modellierung 2018, Braunschweig, Germany, 21-30.

[27] Phoenix contact. RAMI 4.0 and IIRA reference architecture models - A question of perspective and focus, from https://www.mynewsdesk.com/material/document/56241/download?resource type=resource_document, accessed October 15, 2018.

[28] VDI/VDE-Gesellschaft (2015). VDI/VDE-Gesellschaft Mess- und Automatisierungstechnik, Status report - Reference Architecture Model Industrie 4.0 (RAMI4.0), from https://www.zvei.org/fileadmin/user upload/Presse und Medien/Publikationen/2016/januar/GMA Status Repor $t$ Reference Archtitecture Model Industrie 4.0 RAMI 4.0/GMA-Status-Report-RAMI-40-July-2015.pdf, accessed September 15, 2018.

[29] Ulrich, E., Bangemann, T., Bauer, C., Bedenbender, H., Diesner, M., Elmas, F., Friedrich, J., Goldschmidt, T., Göbe, F., Grüner, S., Hankel, M., Heidel, R., Hesselmann, K., Hüttemann, G., Kehl, H., Löwen, U., Pfrommer, J., Schleipen, M., Schlich, B., Usländer, T., Westerkamp, C., Winter, A., Wollschlaeger, M. (2016). Industrie 4.0 - technical assets: basic terminology concepts, life cycles and administration models, Status report, VDI/VDE-GMA, Düsseldorf, Germany, (Permalink: http://publica.fraunhofer.de/documents/N-432116.htmI).

[30] Pisching, M.A., Pessoa, M.A.O., Junqueira, F., dos Santos Filho, D.J., Miyagi, P.E. (2018). An architecture based on RAMI 4.0 to discover equipment to process operations required by products, Computers \& Industrial Engineering, Vol. 125, 574-591, doi: 10.1016/j.cie.2017.12.029.

[31] Johnston, S.J., Basford, P.J., Perkins, C.S., Herry, H., Tso, F.P., Pezaros, D., Mullins, R.D., Yoneki, E., Cox, S.J., Singer, J. (2018). Commodity single board computer clusters and their applications, Future Generation Computer Systems, Vol. 89, 201-212, doi: 10.1016/j.future.2018.06.048. 


\section{APEM}

\title{
Nov model arhitekture za pametno proizvodnjo: Analiza zmogljivosti in primerjava z referenčnim modelom RAMI 4.0
}

\author{
Resman, M. ${ }^{a,}{ }^{,}$, Pipan, $M{ }^{a}{ }^{a}$, Šimic, $M{ }^{\circ}{ }^{a}$, Herakovič, N. ${ }^{a}$ \\ ${ }^{a}$ Department of Manufacturing Technologies and Systems, Faculty of Mechanical Engineering, University of Ljubljana, \\ Ljubljana, Slovenia
}

\section{POVZETEK}

$\mathrm{V}$ tem prispevku smo predlagali nov arhitekturni model pametne tovarne, ki bo proizvajalcem omogočil lažje in natančnejše načrtovanje novih pametnih tovarn z uporabo vseh ključnih tehnologij industrije 4.0. Obstoječi kompleksni referenčni arhitekturni model industrije 4.0 (RAMI 4.0) ponuja dober pregled nad arhitekturo pametne tovarne, vendar vodi do nekaterih omejitev in premalo jasnosti za uporabnike. Za premagovanje teh omejitev smo razvili preprost model s celotno in zelo preprosto arhitekturo pametne tovarne, ki temelji na konceptu porazdeljenih sistemov z natančnimi informacijami in pretokom podatkov med njimi. Predlagani arhitekturni model omogoča bolj zanesljivo in preprosto modeliranje pametne tovarne kot obstoječi model RAMI 4.0. Naš pristop izboljšuje obstoječo metodologijo za načrtovanje pametne tovarne in naredi vse potrebne korake jasnejše. Na koncu prispevka je bila narejena primerjava predlaganega arhitekturnega modela LASFA (LASIM Smart Factory) z obstoječim modelom RAMI 4.0. Razvit model LASFA je bil že uspešno implementiran $\mathrm{v}$ laboratorijskem okolju za gradnjo demonstracijskega centra pametne tovarne.

\section{PODATKI O ČLANKU}

Ključne besede: Industrija 4.0;

Pametna proizvodnja;

Pametna tovarna;

Arhitekturni model;

Referenčni arhitekturni model; RAMI 4.0

*Kontaktna oseba: matevz.resman@fs.uni-lj.si (Resman, M.)

Zgodovina članka:

Prejet 20. decembra 2018

Popravljen 24. aprila 2019

Sprejet 7. maja 2019 